\title{
La production du renseignement comme système d'action
}

Jérôme Marchand

\section{(2) OpenEdition}

12 Journals

Édition électronique

URL : http://journals.openedition.org/communicationorganisation/2124

DOI : 10.4000/communicationorganisation. 2124

ISSN : $1775-3546$

Éditeur

Presses universitaires de Bordeaux

Édition imprimée

Date de publication : 1 novembre 1998

ISSN : 1168-5549

Référence électronique

Jérôme Marchand, "La production du renseignement comme système d'action », Communication et organisation [En ligne], 14 | 1998, mis en ligne le 26 mars 2012, consulté le 19 avril 2019. URL : http:// journals.openedition.org/communicationorganisation/2124; DOI : 10.4000/

communicationorganisation. 2124

Ce document a été généré automatiquement le 19 avril 2019

(c) Presses universitaires de Bordeaux 


\title{
La production du renseignement comme système d'action
}

\author{
Jérôme Marchand
}

1 Si les recherches menées à l'heure actuelle dans le champ de l'intelligence économique font une large place aux technologies de l'information et aux pratiques de veille concurrentielle, l'étude des systèmes d'interactions liant collecteurs de données et analystes, d'un côté, analystes et décideurs stratégiques, de l'autre, semble quelque peu délaissée. Ce qui ne manque pas de surprendre, dans la mesure où l'optimisation des ressources informationnelles dépend, en dernier ressort, de la qualité des circuits de communication liant les uns aux autres ces acteurs (Lesca et Lesca, 1995). Mais comment appréhender les échanges complexes qui s'établissent à l'occasion de la production et de la mise en circulation d'informations à haute valeur ajoutée ? L'une des manières les plus usuelles de répondre à cette interrogation consiste à faire appel à la notion de « cycle du renseignement ", érigée en modèle par les analystes de la C.I.A. ${ }^{1}$ voici près d'un demisiècle, et aujourd'hui plébiscitée par les spécialistes français de l'intelligence économique: enseignants, administrateurs ${ }^{2}$, consultants, journalistes, fabricants de moteurs de recherche ${ }^{3}$, espions en quête de reconversion... Cette greffe, pour autant, a-telle pris? Sans aller jusqu'à parler de «fétichisation » et de « réification » du concept, rares sont les utilisateurs qui osent adopter une démarche dynamique, consistant à intégrer le modèle dans leurs théories et leurs enquêtes, pour lui donner une inflexion propre et renrichir ${ }^{4}$. A un modeste niveau, la présente contribution se propose d'amorcer le débat, en évoquant à grands traits l'expérience de la C.I.A., et en prenant appui sur les réflexions critiques formulées par les élites politiques de Washington et par les chercheurs spécialisés opérant dans le champ du renseignement d'État.

\section{Une notion aux origines incertaines}

2 Quoique la notion de "cycle du renseignement" possède une histoire distincte, les circonstances précises dans lesquelles ce modèle a intégré le répertoire conceptuel de la C.I.A. baignent, aujourd'hui encore, dans le flou ${ }^{5}$. Il en est fait mention dès 1949 , dans un 
ouvrage de Sherman Kent portant sur la place du renseignement dans le dispositif stratégique US, mais sur un mode elliptique (Kent, 1949). Une description plus fouillée du modèle figure dans le premier ouvrage de Harry Howe Ransom, Central Intelligence and National Security, publié à la fin de la décennie suivante (Ransom, 1958). Précisons toutefois que, dans un cas comme dans l'autre, le propos central de l'auteur n'était pas tant d'analyser les logiques et les techniques de production du renseignement que de marquer l'existence d'un champ de compétence autonome, voué à « élargir l'horizon du savoir» (Kent, 1949, p.155-156) et à améliorer le contenu des décisions politicoadministratives. Bref, ainsi compris, le modèle se présente à l'origine comme un instrument rhétorique de légitimation, dont la finalité à peine voilée consistait à justifier les ambitions de la C.I.A.

3 A partir des jalons posés par S. Kent et H.H. Ransom, la notion de «cycle du renseignement " a par la suite fait l'objet d'innombrables déclinaisons. Cependant, abstraction faite des variations terminologiques et du type de découpage séquentiel retenu $^{6}$, les modèles diffèrent assez peu les uns des autres. Celui que diffuse à l'heure actuelle la C.I.A. (CIA, n.d., p. 6-7 ; CIA, 1993, p. 10-11; document Internet) s'articule en cinq phases: planification et direction, collecte, traitement, analyse et production, et enfin dissémination. L'on peut en reprendre brièvement les principaux éléments constitutifs :

4 - La première phase du processus se déclenche lorsque les décideurs (civils ou militaires), étiquetés sous le label de "consommateurs", sollicitent les services spéciaux pour répondre à un besoin informationnel. Selon les cas, ce besoin s'exprime sur un mode ponctuel ou sous la forme d'une liste de questions.

5 - Les échelons supérieurs des instances ainsi sollicitées (i.e., les "producteurs») convertissent ces demandes, leur donnent un format adapté, allouent des ressources aux responsables en charge de la collecte, et déterminent l'orientation globale des efforts organisationnels.

6 - Les unités en charge de la collecte identifient leurs cibles, obtiennent les données brutes requises, et intègrent ces dernières dans leurs rapports. Deux types de précisions s'y ajoutent: les unes ayant trait à la fiabilité des sources mises à contribution, les autres portant sur les conditions dans lesquelles s'est opéré le recueil d'informations.

7 - Une fois centralisée, la masse d'informations ainsi recueillie est transmise aux analystes, qui la convertirent en "produit raffiné » (i.e, le renseignement proprement dit). Ramenée à l'essentiel, la fonction analytique consiste à mettre en balance données récentes et stocks de données antérieures, à faire ressortir la signification et la portée du matériau traité, et à dresser un tableau prospectif. Le tout sans omettre de dégager le degré d'incertitude s'attachant à telle ou telle dimension de la question traitée, de façon à ne pas tomber dans le piège du rehaussement cognitif $^{7}$ et de l'auto-validation des choix préférentiels.

8 - En fin de cycle, analyses et estimations sont distribuées à leurs destinataires, sous des formes variées, à la fois adaptées aux niveaux de compétence et d'habilitation de la clientèle et calibrées en fonction des problèmes traités. Pour reprendre la typologie élaborée par Sherman Kent (Kent, 1949, p. 7-8), les produits du cycle du renseignement se répartissent en trois catégories dominantes: "current reportorial» (questions d'actualité), «basic descriptive » (fonds documentaires), et "spéculative-évaluative » (estimations à forte charge prospective). 
9 En fonction des résultats obtenus et des réactions enregistrées, de nouvelles interrogations et de nouvelles demandes viennent s'inscrire sur l'agenda de recherche des spécialistes du renseignement et de leurs interlocuteurs. Le cycle se réamorce.

10 Ainsi résumé, l'ensemble parait à bien des égards influencé par les schémas de rationalité ayant cours dans les forces armées. De là à suggérer que le cycle du renseignement a pris naissance dans le monde du renseignement militaire, il n'y a qu'un pas. Dans un ouvrage paru voici deux ans et immédiatement promu au rang de classique, Michael Herman a relevé quelques-uns des indices susceptibles d'étayer cette hypothèse: "simplification des processus complexes, séquences linéaires standard; et importance des exercices de discipline mentale » (Herman,1996, p. 287). Dans la même direction, l'on peut ajouter ici que ces composantes procèdent toutes d'un schéma d'organisation commun, fondé sur le principe de l'autorité hiérarchisée, et dont la prégnance se manifeste en priorité sur le terrain des modes d'articulation entre consommateurs et producteurs de renseignements. Telle qu'elle se présente dans l'approche "séquentielle-linéaire " détaillée ci-dessus, l'expression des besoins informationnels se trouve appréhendée comme émanant d'un acteur unique, apte à se déterminer en fonction de l'objectif qu'il s'est choisi et capable de suivre un cheminement rectiligne, allant de la formulation de la requête initiale à l'évaluation finale des produits («outputs»). En ce sens, la métaphore du cycle du renseignement se rattache au modèle traditionnel de la décision cartésienne.

11 Selon toute vraisemblance, une telle filiation idéologique n'est en rien le fruit du hasard. Rappelons en effet que les autorités US, à partir de la fin des années cinquante, se lancèrent dans un programme d'optimisation des ressources et de rationalisation des choix administratifs, faisant une large place aux méthodes PPBS $^{8}$. Que la CIA. ait cherché à s'inscrire dans ce mouvement, dans le but d'asseoir sa légitimité et d'élargir sa base d'influence, semble plausible. Revers de la médaille, cet infléchissement a eu pour effet de reléguer dans l'ombre un certain nombre de questions fondamentales: sur quelles croyances l'autorité des décideurs politico-administratifs s'appuie-t-elle? De quels moyens l'homme de pouvoir dispose-t-il pour assurer et maintenir sa prééminence vis-àvis des spécialistes du renseignement? Comment peut-il amener les services spéciaux à traiter ses requêtes informationnelles en temps utile et dans le sens désiré ? Au moyen de quels instruments peut-il évaluer la qualité des informations et des analyses qui lui sont transmises? Dans leur analyse des processus de production du renseignement, les tenants de l'approche séquentielle-linéaire ont d'emblée cédé à une vision naturaliste, d'où était exclue toute réflexion poussée sur les schémas d'influence réciproque à même de se nouer entre décideurs politiques et spécialistes du renseignement. A bien des égards, la C.I.A. a dû payer le prix de cette omission, lorsqu'elle a vu se déchirer le voile de confidentialité qui, depuis ses débuts, la protégeait, et lorsque ses fiascos analytiques se sont trouvés portés à la connaissance du grand public.

\section{Le cycle du renseignement à l'épreuve de la controverse}

12 Longtemps exclues de l'aire de débat public, les missions, les techniques opérationnelles et les ressources de l'appareil de renseignement américain ont inspiré, tout au long des années soixante-dix et quatre-vingt, une série de mobilisations à forte charge conflictuelle (Johnson, 1996), dont les effets se font encore sentir à l'heure actuelle. Axées 
à l'origine sur les pratiques déviantes de la CIA. et de ses congénères, ces réactions de contrôle social n'ont pas tardé à sortir de ce cadre, pour se recentrer sur les processus de production du renseignement et sur leurs modes de représentation. A cette occasion, le cycle du renseignement a vu sa pertinence directement remise en cause. Y compris par les élites politiques de Washington.

13 7. Comprendre le processus de désacralisation dont a fait l'objet le cycle du renseignement exige, en premier lieu, de s'attacher aux critiques officielles formulées dans le prolongement immédiat du Watergate. Sont ici visés, en priorité, les travaux du comité Church'. Du rapport d'enquête publié par cet organe d'investigation parlementaire se détachent deux passages dignes d'attention. Le premier vise le clivage opposant les représentations idéal-typiques fixées par le label « intelligence cycle », d'un côté, et les schémas opérationnels en vigueur dans le champ du renseignement: «en réalité, le schéma est à peine reconnaissable (...). Les requêtes reflètent ce que les managers croient être les besoins des consommateurs, et ce que leurs organisations sont selon eux capables de produire.

Du fait qu'il y a pléthore de managers et que le contrôle central est faible, chacun se trouve à peu près libre de fixer son propre programme de recherche » (U.S. Senate, 1976, p.18). Le second point, complémentaire du précédent, porte quant à lui sur la paupérisation des fonctions analytiques induite par la déliquescence des normes bureaucratiques de responsabilité objective et l'abaissement des barrières entre monde politique et communauté du renseignement: "Les tâches de production du renseignement d'actualité - l'analyse au jour le jour des événements contemporains et sa dissémination rapide - accaparent aujourd'hui le gros des ressources de la DDI ${ }^{10}(. .$.$) .$ L'analyse approfondie des problèmes dotés d'implications à long terme pour les décideurs a reçu moins d'attention et moins de ressources » (U.S. Senate, 1976, p. 272-273).

Construits en opposition explicite aux schémas formels de production du renseignement jusqu'alors diffusés par la C.I.A., les deux points mentionnés ci-dessus visaient de toute évidence à légitimer la réaction de contrôle social animée par les représentants du Congrès. Leur apport, toutefois, ne saurait être appréhendé en termes exclusivement instrumentaux. Avec la remise en question des postulats traditionnels assignant aux spécialistes du renseignement et aux décideurs politiques une vision et des intérêts par essence communs, s'est en effet opéré un renouvellement marqué des schémas d'analyse relatifs à la déviance des services spéciaux. A une approche fixée sur les décideurs politico-administratifs, encline à placer les écarts de la C.I.A. sur le compte de son étatmajor et sur celui de l'Exécutif, s'est de la sorte substituée une nouvelle approche, désignant les scandales survenus dans le champ du renseignement comme la manifestation de vulnérabilités structurelles et de contraintes hautement sectorialisées. En retour, les schémas traditionnels touchant la production et la dissémination du renseignement ont vu leur aura décroître.

\section{La C.I.A. face à la révolution des mollahs}

16 Toujours dans la même perspective, les études de cas consacrées aux déboires analytiques de la C.I.A. ont permis de mieux comprendre le jeu complexe qui lie producteurs et consommateurs de renseignements, au jour le jour, et les multiples distorsions qui en résultent. Une place particulière, dans cette perspective, doit être réservée à la révolution des mollahs. Rappelons brièvement les faits. Début 1978, alors que le régime du Shah était 
en butte à une vague de contestation montante, la Maison Blanche sonda la communauté du renseignement US, afin de mieux cerner la crise et ses lignes d'évolution potentielles. Et parce qu'elle sous-estimait la force de l'Islam politique dans le contexte iranien, et parce qu'elle surestimait les ressources coercitives - police, armée et services secrets placées à la disposition des autorités, la C.I.A. choisit d'entrée une grille de lecture désignant la position du Shah comme stable, à moyen terme. Pire encore, elle se montra incapable de produire en temps utile un National Intelligence Estimate ${ }^{11}$, reflétant les vues globales de la communauté du renseignement US sur la situation en Iran (House Select Committee on Intelligence, 1979). Faute d'avoir su préserver son capital de crédit, la C.I.A. ne fut dès lors plus en mesure de se faire entendre, une fois ses prévisions ajustées. Quant à la situation politique en Iran, elle bascula rapidement. Après une période de relative accalmie estivale, l'agitation reprit de plus belle au cours de l'automne et de l'hiver 1978. Abandonné par ses alliés politiques, le Shah finit par prendre le chemin de l'exil, le 16 janvier 1979.

17 S'appuyant sur les témoignages laissés par les dirigeants politico-administratifs alors en place, les travaux historiques consacrés à l'ère Carter ont longtemps soutenu que, dans cet épisode, la Maison Blanche avait vu sa marge de manœuvre compromise par les défaillances analytiques de la CIA. Faute d'avoir reçu en temps utile un tableau synthétique, fidèle et réaliste, des événements et de leurs lignes d'évolution potentielles, laissait entendre l'historiographie orthodoxe, le pouvoir politique n'avait pas été en mesure de développer une gestion de crise rationnelle et efficace. De toute évidence, ces éléments d'appréciation contiennent une part de vérité. Dans son rapport final, la commission d'enquête parlementaire créée spécialement pour faire la lumière sur les événements n'a pas manqué d'identifier plusieurs points de blocage - archaïsme des modèles prévisionnels, inadaptation des circuits d'information locaux, diversification insuffisante des relais de transmission, fermeture cognitive des centres d'analyse, survalorisation des contraintes géostratégiques... -, ensuite convertis en instruments d'étiquetage négatif par les adversaires de la C.I.A. et par divers observateurs indépendants (Laqueur, 1993, p. 136-137).

18 Par chance, depuis la fin de la Guerre froide, les documents déclassifiés en provenance des instances gouvernementales et de l'appareil de renseignement US se font plus nombreux (C.I.A., 1996). Grâce à ce mouvement d'ouverture, les chercheurs ont été en mesure de réévaluer les performances analytiques de la C.I.A. et du State Department. Une place particulière, dans cette perspective, doit être réservée à Michael Donovan, auteur d'une recherche doctorale centrée sur la production du renseignement US lors de la révolution iranienne (Donovan, in Andrew et Jeffrey-Jones, 1997, p. 143-163). A partir des rapports et des estimations diffusés entre janvier et septembre 1978, cet auteur a démontré que la communauté du renseignement US, certes convaincue à l'origine de la stabilité à moyen terme du régime impérial, n'en avait pas moins enregistré la prolifération accélérée des signaux d'alerte, puis modifié en conséquence ses estimations. Mais sans pour autant parvenir à briser le carcan des représentations dominantes ayant cours à la Maison Blanche. "En dernière analyse, la politique américaine était dépendante du Shah et, de ce fait, les mauvaises nouvelles n'étaient pas bien accueillies. Les décideurs négligeaient le renseignement qui n'allait pas dans le sens de leur représentation préférentielle de la situation (...). Washington a vu sa gamme d'options limitée par la nature de la relation américano-iranienne et par les manœuvres bureaucratiques, non par le déficit du renseignement » (Donovan, 1997, p. 160). 


\section{Ébranlements conceptuels}

19 A n'en pas douter, les critiques formulées par la classe politique US ont contribué à ébranler les certitudes acquises et à renouveler la réflexion consacrée au cycle du renseignement. En témoignent les observations fragmentaires parues dans la presse à grand tirage et les publications académiques. Mais c'est dans les années quatre-vingt, avec le développement d'une tradition de recherche autonome s'inscrivant dans le sillage des sciences sociales, que les spécialistes ont relancé le débat et pris quelque distance visà-vis du modèle. Certains auteurs, comme l'éminent historien W. Laqueur, sont même allés jusqu'à mettre en doute son bien-fondé. «Le concept même de cycle du renseignement a quelque chose de malheureux, car un cycle dans le langage courant est une orbite ou bien, en thermodynamique, une série d'opérations au terme desquelles la substance originelle se trouve ramenée à son état initial. Dans toute opération de renseignement, le but final consiste à accumuler une masse de connaissances supérieure au capital de départ » (Laqueur, 1993, p. 346).

Poursuivant dans cette direction, le travail de réévaluation systématique mené par les chercheurs n'a pas tardé à se focaliser sur les schémas d'organisation rationnelle censés gouverner la production et la diffusion du renseignement. En ce qui concerne la collecte, tout d'abord, on a assisté à une remise en cause des schémas formels de répartition du travail qui figuraient au cœur du modèle classique élaboré par S. Kent et H.H. Ransom. Qu'il s'agisse de dénoncer le peu d'attention prêté aux schémas de perception et d'interprétation structurant le travail des collecteurs - et donc, par voie de conséquence, celui de leurs collègues analystes situés en aval de la chaîne -, qu'il s'agisse d'attirer l'attention sur les fonctions de régulation assumées par les agences spécialisées dans la collecte SIGINT ${ }^{12}$, ou qu'il s'agisse de marquer la considérable valeur ajoutée s'attachant au renseignement de pénétration par rapport au renseignement d'observation, sociologues, politistes historiens et praticiens n'ont pas manqué de faire ressortir le caractère artificiel et contre-productif des graduations qualitatives et hiérarchiques figurant au cœur du modèle classique, et assignant à la collecte un rang subalterne. Plus systématiquement encore, les évaluations initiales menées par les collecteurs individuels ont fait l'objet d'une réestimation à la hausse, à tel point qu'elles sont aujourd'hui considérées par beaucoup comme partie intégrante du processus analytique.

En parallèle, les réflexions relatives à l'analyse spécialisée ont elles aussi connu un mouvement de renouvellement marqué, mettant en cause le développement omnidirectionnel des foyers d'investigation et l'alourdissement corrélatif des structures et des processus. Dès lors qu'un service de renseignement étend ses ramifications beaucoup plus loin que ne le voudraient la stricte logique capitaliste et la rationalité bureaucratique, la complexité qu'il engendre de la sorte engendrent de multiples surcoûts, ainsi que des réponses inappropriées (retards, erreurs d'aiguillage, dilution conceptuelle, etc.). Largement inspiré par les dérives expansionnistes de la C.I.A., ce constat a conduit les spécialistes à se distancier des logiques monopolistiques latentes alimentées par les idéaux technocratiques et à reconsidérer l'ajustement des pratiques organisationnelles avec les besoins du système global. En termes concrets, il est aujourd'hui admis que l'exigence de rendement impose des mesures telles que l'autoréduction du champ d'investigation, le décloisonnement des réseaux d'expertise, ou encore le court-circuitage partiel de la phase d'analyse en période de crise, lorsque les 
contraintes temporelles se resserrent, en même temps que les risques de déformation des signaux s'amplifient.

Dans le domaine de la dissémination, enfin, une autre avancée conceptuelle importante a pris place grâce à une réinterprétation dynamique des facteurs symboliques susceptibles de favoriser l'injection du renseignement dans les processus décisionnels. En se plaçant dans une perspective rationaliste, l'approche séquentielle-linéaire avait en effet contribué à reléguer à l'arrière-plan l'analyse des stratégies d'influence et de persuasion mises en œuvre par les centrales de renseignement, dans le but de modeler l'environnement domestique où elles évoluent. Or l'expérience montre qu'il s'agit là d'un facteur d'intégration et de légitimation central, étant entendu que ces organisations opèrent en permanence en marge de la légalité et des mythes démocratiques, et se trouvent de ce fait confrontées au quotidien à des logiques d'ostracisme appuyées.

\section{Une adaptation devenue impérative}

Confrontée à ces observations, stimulée par les réflexions critiques en provenance de la classe politique, la C.I.A. a dû s'adapter. Dans la pratique, cette réorientation s'est traduite par un train de réformes allant dans le sens d'une réduction des cloisonnements horizontaux et verticaux, d'une diversification des méthodes d'évaluation, et d'une redistribution des responsabilités, passant par la mise en place de procédures de concertation entre les différents réseaux d'expertise qui sillonnent la communauté du renseignement US. Les échelons dirigeants de la C.I.A., en outre, se sont efforcés de resserrer les liens de la centrale avec ses principaux interlocuteurs politiques. Mais sans pour autant chercher à réactiver la mystique technocratique d'antan. A ce système de représentations quelque peu daté s'est substituée une nouvelle approche inspirée des méthodes ayant cours dans le monde de l'entreprise, et centrée sur la satisfaction immédiate du « consommateur ».

Des micro-ajustements ainsi opérés se détachent quelques points saillants: formatage standard des notes analytiques adressées aux membres du Congrès, multiplication des briefings oraux, exploitation intensive des techniques audio-visuelles (transparents multicolores, cassettes vidéo, CD-roms), sélection affinée des officiers de renseignement chargés d'assurer la liaison avec les instances politico-administratives... (Johnson, 1996). Sans doute de telles dispositions font-elles piètre figure, mesurées à l'aune des estimations stratégiques autour desquelles s'articulent les missions de recherche et de réflexion menées par l'appareil de renseignement US (Ford, 1993). Leur impact cumulatif, pour autant, ne saurait être tenu pour négligeable. Non seulement parce que l'injection du renseignement dans les processus décisionnels s'en est trouvée facilitée. Mais aussi parce que cet effort d'adaptation a contribué à normaliser les relations de la centrale avec ses contrôleurs parlementaires. Qu'il s'agisse de faire acte de présence lors des cérémonies officielles ou qu'il s'agisse d'animer les sessions de travail faisant appel à son expertise, la C.I.A. a appris à tenu-compte du système de contraintes particulièrement pesant dans lequel sont pris les décideurs politiques de Washington ${ }^{13}$. En retour, ces efforts d'adaptation lui ont permis de reconquérir une partie des appuis qui s'étaient volatilisés durant les années soixante-dix et quatre-vingt. 


\section{Un modèle encore valide?}

25 continuant à faire une large place au cycle du renseignement dans leurs travaux, la plupart des spécialistes anglo-saxons, conscients des limites de l'approche traditionnelle, se sont efforcés de renouveler le modèle. Non sans succès, pour certains. C'est le cas, en tout premier lieu, d'Arthur S. Hulnick - officier de renseignement en poste à la C.I.A. durant trente-cinq années ${ }^{14}$ - qui a consacré plusieurs études aux connexions liant consommateurs et producteurs de renseignements et aux mesures susceptibles d'optimiser cette relation (Hulnick, 1987). C'est le cas, plus récemment de Michael Herman, lui aussi doté d'une solide expérience professionnelle dans les milieux du renseignement ${ }^{15}$, et auteur d'un authentique ouvrage de référence mettant en avant la forte dimension organisationnelle de ce champ d'activités ${ }^{16}$ (Herman, 1996). Sans qu'il soit question de reprendre en détail les arguments formulés par ces deux auteurs, quelques points fondamentaux valent d'être mis en avant.

De manière générale, le premier grief formulé à rencontre du modèle classique met en cause son caractère arbitraire et simplificateur. Certes, la notion de cycle du renseignement permet d'identifier quelques-unes des tâches-clés auxquelles sont confrontés les spécialistes des services spéciaux. Mais rend-elle compte du système d'échanges pour le moins complexe qui s'établit entre les différents pôles organisationnels concernés, une fois le processus de production enclenché? Pour A. Hulnick comme pour $\mathrm{M}$. Herman, la réponse à cette interrogation s'avère négative. Loin de se succéder en flux continu, les tâches sectorielles et fragmentaires auxquelles se consacrent les S.R. s'inscrivent dans un ensemble multi-fonctionnel, dont les éléments sont connectés à tous moments et à tous niveaux. De sorte que chaque étape du processus renvoie aux autres étapes. Fort de ce constat, auquel souscrivent aujourd'hui la plupart des spécialistes anglo-saxons ${ }^{17}$, A. Hulnick se trouve conduit à proposer un schéma alternatif, dans lequel l'accent se trouve mis sur la coexistence de rationalités et de procédures interdépendantes : «Il est (...) important de reconnaître que certaines étapes du processus opèrent sur un mode séquentiel, mais que d'autres sont quasi-autonomes. A ce titre, il semble préférable d'envisager le processus comme une matrice d'éléments interconnectés plutôt que comme une séquence de fonctions connectées » (Hulnick, 1987, p. 136).

27 Toujours selon A. Hulnick, l'excès d'abstraction inhérent au modèle classique pose problème par la confusion qu'il introduit entre les différents processus cognitifs à l'œuvre dans les dynamiques de mobilisation organisationnelle. Postulant, à partir d'une conception moniste, l'existence d'une rationalité partagée entre spécialistes de la collecte et spécialistes de l'analyse, les tenants de l'approche linéaire ont laissé de côté la question des perceptions et des stimuli contribuant à activer les différentes séquences du cycle. Or, sur ce point, les témoignages fournis par les professionnels convergent : selon le domaine d'activité, la construction sociale des besoins informationnels varie dans des proportions parfois considérables. «Les exigences de la collecte et celles de l'analyse sont tout à fait différentes, observe A. Hulnick. D'habitude, la première de ces deux phases se trouve activée lorsque l'on découvre des vides dans les banques de données, tandis que la seconde d'entre elles peut fort bien se déclencher en réponse à une situation de crise exigeant, quelque part dans le monde, une réaction politique (...) Les demandes formulées 
dans le domaine de la collecte proviennent le plus souvent d'analystes qui appréhendent les insuffisances informationnelles ou bien des collecteurs eux-mêmes, parce qu'ils suivent la progression logique de l'information, au fur et à mesure qu'ils la recueillent, et parce qu'ils savent ce qu'il faudrait engranger par la suite. Les requêtes formulées par les décideurs portent le plus souvent sur l'analyse, la recherche ou l'évaluation de l'information » (Hulnick, 1987, p. 136-137). En ce sens, la production du renseignement n'est pas simplement production d'informations validées à usage fonctionnel. Elle se présente également comme la production de signes et de symboles répondant à des codes culturels distincts, en fonction et au moyen desquels se dégagent et se hiérarchisent les besoins informationnels des différents intervenants. Maîtriser ces codes constitue donc une priorité, dès lors que l'on prétend optimiser le fonctionnement des centrales de renseignement.

\section{Des logiques de production diversifiées}

De même que la genèse des besoins informationnels, la structuration des échanges entre " producteurs » et " consommateurs » de renseignements a, elle aussi, fait l'objet d'un travail de réévaluation poussé. Dans sa formulation classique, le cycle du renseignement répondait à une vision stratégique assignant aux décideurs politico-administratifs un dessein concret, sous-tendu par des motivations rationnelles, et implémenté au moyen d'instruments adaptés. Les témoignages livrés par les spécialistes du renseignement montrent que cette vision ne correspond pas à la réalité. Qu'il s'agisse des biais cognitifs propres aux décideurs, des limites frappant l'information accessible, de la faillibilité des instruments d'analyse disponibles, de multiples variables nuisent à la qualité des flux de guidage. Plus encore, selon A. Hulnick et M. Herman, les limites de l'action rationnelle tiennent aux logiques de production différentielles à l'œuvre dans le champ du renseignement. Contrairement à ce que laissaient entendre les travaux de S. Kent et de H.H. Ransom, les schémas d'articulation entre centrales de renseignement et décideurs ne peuvent être traités de manière abstraite et globalisante. Le renseignement de « mise en alerte ("warning intelligence») obéit à de toutes autres contraintes que le renseignement prospectif («estimative intelligence ») ou le renseignement d'actualité ( current intelligence »).

Ces mêmes schémas, en outre, voient leur axe directionnel varier du tout au tout en fonction du volume de données recueilli et traité. Ainsi que M. Herman le fait valoir de manière convaincante, le schéma du décideur unique procédant «à la carte ", assignant aux services spéciaux le soin de répondre à telle ou telle interrogation, n'est valide que dans une optique artisanale. Dès l'instant où la palette de missions confiée à l'appareil de renseignement s'universalise, dès lors que l'on bascule dans une logique de production de masse, faisant une large place à la technologie moderne (instruments de collecte satellites, stations d'écoute - et de traitement - centres de décryptage -), les rôles s'inversent. L'initiative doit revenir aux centrales de renseignement : « Les producteurs à grande échelle (...) constituent la force motrice, c'est à eux de recueillir un feed-back auprès des utilisateurs et de l'exploiter pour parvenir à une optimisation des produits. Une unité de production efficace explore constamment de nouveaux sujets et expérimente de nouvelles techniques de mise en forme, à la recherche de ce que les Américains désignent comme «le renseignement sur mesure " ( tailored intelligence »), adapté aux besoins particuliers des consommateurs. (...) L'objectif du renseignement est 
de faire en sorte que le produit reçu par l'usager déclenche en lui une satisfaction maximale »(Herman, 1996, p. 293).

\section{Réhabilitation de la prospective}

A ce renversement dans l'approche théorique des modes de production du renseignement correspond, par force, une série de déplacements touchant les logiques de fonctionnement organisationnel. Deux points, dans cette optique, méritent de recevoir une attention particulière. Le premier concerne la fonction prospective-spéculative. Dès lors que l'on cesse d'opérer dans un cadre rationaliste et globalisant, la nécessité de procéder à un travail de réflexion prospective se fait sentir avec une acuité accrue. Faute de directives cohérentes susceptibles de fixer et de hiérarchiser les priorités organisationnelles, la production du renseignement acquiert par force un caractère spéculatif plus marqué. Au lieu de se fixer sur la recherche de «secrets» (militaires, politiques, économiques, industriels...) dérobés aux yeux du commun, la mission première des organes de renseignement se recentre sur l'exploration du champ des possibles, à la manière d'un «think-tank ». Mais sans pour autant procéder à l'aveuglette ou se caler sur les courants de recherche innervant le monde universitaire. Comme l'a fait valoir voici quelques années Roy Godson (Godson, 1989, p. 5-8), comme le souligne à son tour Michael Herman (Herman, $19 \%$, p. 229), une telle réorientation ne fait sens que si 1) les services concernés se révèlent aptes ${ }^{18}$ à pratiquer un renseignement analytique d'opportunité, en phase à la fois avec les objectifs pérennes de la collectivité nationale et les priorités changeantes du leadership gouvernemental, et 2) s'ils savent par ailleurs s'adapter aux perturbations environnantes ${ }^{19}$. Cette double condition se trouvant remplie, la fonction des centrales de renseignement consiste alors à ébaucher des axes de réflexion, à indiquer des pistes, à élaborer des scénarios alternatifs pour affronter les crises et les ruptures en gestation, de manière à réintroduire un semblant d'ordre dans la complexité environnante et à offrir aux élites dirigeantes une marge de réflexion spéculative.

Corollaire du point précédent, un service s'engageant sur la voie du renseignement d'opportunité ne se montrera efficace que s'il est tenu informé - de manière précise, rapide et régulière - des réponses et des réactions de ses interlocuteurs politicoadministratifs, et de l'effet des données injectées dans le processus décisionnel. Procéder autrement, c'est rendre inéluctables la domination du court terme et le fractionnement des tâches dévolues aux services spéciaux ${ }^{20}$. Fort de ce constat, auquel souscrit également A. Hulnick (Hulnick, 1987), M. Herman se trouve ainsi conduit à prôner le renforcement des dispositifs de correction et de réglage, de façon à assurer un ajustement permanent de l'action organisationnelle aux réactions de l'environnement de contrôle. Plus encore, le spécialiste britannique va même jusqu'à ébaucher un système d'interactions confiant aux centrales de renseignement un rôle de "catalyseur". Par opposition au modèle classique, qui réservait l'initiative - ou ses apparences - aux décideurs, l'on débouche de la sorte sur un système d'échanges dans lequel le rôle des producteurs de renseignements consiste à lancer des coups de sonde en direction de leurs interlocuteurs, à identifier les points qui font réagir les consommateurs - effectifs / virtuels - visés, puis, ceci fait, à explorer sans délai les axes d'investigation et de réflexion ainsi ébauchés. «Dans le cycle du renseignement ainsi ajusté, note M. Herman, le renseignement se trouve placé en position de contrôle et les réactions de l'usager font office d'input primaire » (Herman, $19 \%$, p. 234). Avec à la clé un risque majeur, ajouterons-nous : celui de voir l'appareil de 
renseignement fausser les termes de l'échange en essayant - par simulation et projection - d'anticiper les comportements de ses interlocuteurs politico-administratifs et les conséquences de leurs choix.

\section{Conclusion}

32 A en juger par la production scientifique récente, les déplacements amorcés au cours des années quatre-vingt n'ont pas encore abouti au changement de perspective annoncé. Même si les chercheurs universitaires ont pris conscience des limitations frappant le cycle du renseignement (Johnson, 1996, p. 213), même si les professionnels eux-mêmes se disent convaincus de la nécessité de faire évoluer les schémas de représentation en vigueur dans leur propre milieu, les uns et les autres n'en continuent pas moins à faire un large usage de cet instrument. Son apport, il est vrai, a été et reste capital. Et ce pour au moins trois raisons. Le cycle du renseignement, tout d'abord, a permis de rompre avec l'empirisme tâtonnant qui a longtemps caractérisé les ouvrages traitant des services spéciaux, de leurs activités, de leurs logiques d'organisation, et de leurs interactions avec l'environnement extérieur. L'approche ainsi proposée, en outre, se veut dynamique. Elle rompt avec les pseudo-abstractions géopolitiques, riches en stéréotypes, auxquelles succombent si fréquemment les spécialistes $\mathrm{du}$ renseignement et leurs relais journalistiques. Enfin elle démystifie le renseignement. Loin d'autonomiser les activités des S.R., elle les replace dans un cadre systémique. Avec ce type d'approche, les appareils de renseignement valent par ce qu'ils produisent effectivement, non par leurs schémas d'auto-identification proto-mythologiques. A l'heure où tant de "spécialistes » en sécurité économique restent prisonniers de logiques paranoïaques d'un autre âge, et sans grande portée pratique ${ }^{21}$, le point vaut d'être souligné.

\section{BIBLIOGRAPHIE}

Central Intelligence Agency, Intelligence, the acme of skill, Washington D.C., non daté.

C.I.A., Fact book on intelligence, Washington D.C., C.I.A., 1993.

DONOVAN Michael, National intelligence and the iranian revolution, in Eternal Vigilance? Fifty years of the C.I.A., Londres, Frank Cass, 1997, p. 143-163.

FORD Harold P., Estimative intelligence : the purposes and problems of national intelligence estimating, Boston, University press of America, 1993.

GODSON Roy (ed.), Intelligence requirements for the 1990s, Lexington, Lexington Books, 1989.

HERMAN Michael, Intelligence power in peace and war, Cambridge, Cambridge university press, 1996.

HULNICK Arthur S., " Relations between intelligence producers and policy consumers : A new way of looking at an old problem », in Cimbala Stephen J. (ed.), Intelligence and intelligence policy in a democratic society, Dobbs ferry, New York, transnational, 1987. 
JOHNSON Loch K., Secret agencies : U.S. intelligence in a hostile world, Yale, Yale university press, 1996.

KENT Sherman, Strategic intelligence for world policy, Princeton, Princeton university press, 1949.

LAQUEUR Walter, The uses and limits of intelligence, New York, Basic Books, 1993.

LESCA Humbert et Lesca Élisabeth, Gestion de l'information. Qualité de l'information et performances de l'entreprise, Paris, Litec, 1995.

RANSOM Harry Howe, Central intelligence and national security, Cambridge, Harvard university press, 1958.

U.S. Congress, Senate, Select committee to study governmental operations with respect to intelligence activities (The Church committee), Final report, 94 th Congress, 2nd session, Washington, DC, U.S. G.P.O., 1976.

\section{NOTES}

1. Central Intelligence Agency.

2. Levet Jean-Louis, «L'intelligence économique : fondements méthodologiques d'une nouvelle démarche » in Revue d'Intelligence Économique, n 1, mars 1997, p. 42.

3. Entretien avec Patrice Allard / société Datops. Paris, juillet 1997.

4. Dans cette direction, voir les propositions de Stevan Dedijer. Entretien publié dans Veille, $\mathrm{n}$ - 17, septembre 1996, p. 3-5.

5. Entretien avec le Pfr. Kenneth Mac Donald, ancien historien en chef de la C.LA. Luc-sur-Mer, 8 février 1998.

6. A titre indicatif, les modèles les plus rudimentaires reposent sur un découpage ternaire : collecte, analyse, dissémination.

7. « Cognitive bolstering ».

8. Planning Programming Budgeting System.

9. U.S. Senate, Select Committee to Study Governmental Operations with respect to Intelligence Activities («Church Committee »).

10. Directoire de l'analyse.

11. Document de synthèse exprimant les vues de la communauté du renseignement tout entière.

12. Signal Intelligence.

13. Sur l'accesion de la CIA au rang d'arbitre dans le conflit israélo-palestinien, voir International Herald Tribune, 21 octobre 1998.

14. Et aujourd'hui reconverti dans le champ de la «business intelligence ». Le Pfr. Hulnick est membre de la SCIP-USA et de l'ASIS

15. Mais sur l'autre rive de l'Atlantique, puisqu'il fit carrière dans le service d'interceptions britannique (General Communication Headquarters) et anima l'une des cellules de coordination du renseignement opérant à Whitehall

16. Voir le compte-rendu à paraître in Politique Étrangère, décembre 1998

17. Leurs homologues français, quant à eux, restent attachés au modèle séquentiel-linéaire. Dans ce sens, voir l'article de Christian Malis, « Renseignement et Information », in L'Armement - Revue de la DGA. $N^{\circ} 60$, décembre 1997-janvier 1998, dans lequel le cycle du renseignement se trouve défini comme un processus «continu» (p.29). A noter toutefois que l'auteur opère dans le domaine du renseignement militaire (Direction du Renseignement Militaire), secteur dont les contraintes et les logiques d'action diffèrent assez sensiblement de celles propres au monde du renseignement civil et stratégique 
18. Le., politiquement éclairés, donc libres d'allégeances idéologiques, partisanes, factionnelles trop marquées et trop pesantes. Selon le Pfr Douglas Porch, l'appareil de renseignement extérieur français (SDECE.DGSE) n'est jamais parvenu, depuis la Seconde Guerre, à s'émanciper. Et ce, notamment, parce qu'il s'agit d'un service placé sous la coupe du monde militaire et dépourvu d'une tradition analytique forte. Entretien avec D. Porch, Agneaux, 23 novembre 1997.

19. Sur ce terrain, les $S R$. français semblent jouir d'une réputation honorable. Entretien téléphonique avec le lieutenant-colonel Jacques F. Baud -ancien officier du renseignement helvétique - juillet 1997.

20. L'expérience de la Cinquième République, a cet égard, est parlante

21. Entretien avec S Dedijer, in Veille. $n^{\circ} 17$, p. 3-5.

\section{RÉSUMÉS}

Conçu par les analystes de la C.I.A. voici près de cinquante ans, le cycle du renseignement jouit d'une grande popularité dans les milieux de l'intelligence économique, version française. Critiqué outre-Atlantique pour son caractère quelque peu mécanique et artificiel, ce modèle a fait l'objet d'amendements répétés, qui ont contribué à renouveler l'étude des rapports entre consommateurs et producteurs de renseignements.

The intelligence cycle, recently appropriated by French economic intelligence specialists, was first developed by C.I.A. analysts half a century ago, to build their own basis of influence. Over the last twenty-five years, various voices have challenged the value of this instrument and forced the U.S. intelligence community to alter its own view of intelligence-policy relationships. Yet, although periodic flurries of criticism lead to occasional reformulations, the model remains widely used.

\section{INDEX}

Mots-clés : cycle du renseignement, collecte, analyse, dissémination, Central Intelligence Agency

\section{AUTEUR}

\section{JÉRÔME MARCHAND}

Jérôme Marchand est chercheur associé auprès de l'Institut des Hautes Études Judiciaires ; co-animateur du module d'enseignement « The Enactment of Strategic Intelligence » (HEC) ; membre du groupe de recherche « Modernité de l'État » (Fondation Saint-Simon) ; membre de « Transparency International » (chapitre français). Ses axes de recherche sont : l'information « sensible » et sa gestion en milieu organisationnel (renseignement d'État, intelligence économique, lutte anti-corruption, scandales politico-adiministratifs, communication de crise). 